\title{
UTILIZANDO A METODOLOGIA PDCA PARA A MELHORIA DO PROCESSO DA CONSTRUÇÃO DE UMA PONTE DE ESPAGUETE
}

DOI: 10.37702/2175-957X.COBENGE.2021.3464

CLAUDIO BONFANTE DE OLIVEIRA - claudiobonfa@yahoo.com.br CORPO DE BOMBEIROS MILITAR DO ESTADO DO RIO DE JANEIRO Rua João Fabricio José 195 25812-030 - Três Rios - RJ

Arthur de Souza Carvalho - arthurscarvalho@hotmail.com Marcom Terraplenagem Rua Maestro Costa Barros 717 25805-090 - Três Rios - RJ

Luis Otavio de Oliveira Pancotti - tavinhoconstruir@gmail UNIVERSIDADE DE VASSOURAS

R RITA DE JESUS MOURA DOS SANTOS 96 27700-000 - VASSOURAS - RJ

Larissa Leal Coutinho - lariissasouza87@gmail.com Compainha Brasileira de Soluções em Engenharia LTDA Av. Marechal Paulo Torres 952 27700-000 - Vassouras - RJ

Carlos Vitor de Alencar Carvalho - cvitorc@gmail.com Centro Universitário Estadual da Zona Oeste e Universidade de Vassouras Rua General Belegarde 185 20710-003 - Rio de Janeiro - RJ

Resumo: Este trabalho tem o objetivo de mostrar resultados experimentais, a partir de uma pesquisa realizada na Universidade de Vassouras e analisar os resultados obtidos na elaboração de uma ponte de espaguete, utilizando o método PDCA como instrumento de melhoria na elaboração e execução do projeto. A avaliação foi executada através de testes de compressão e tração no laboratório da Universidade de Vassouras. Iniciou-se a execução e durante o processo foi identificado vários problemas tanto nos materiais como no método de execução ,onde para os materiais foram sendo submetidos a um controle de qualidade 
sendo descartados os despadronizados e na execução utilizou-se gabaritos para a montagem, com as medidas pré estabelecidas pelo professor, durante todo o processo a melhoria foi sendo feita observando-se o resultado no teste de esforço e assim foi sendo aperfeiçoado todo o processo eliminando possíveis erros até que fosse adquirido o resultado definido no projeto.

Palavras-chave: PDCA, Ponte de Espaguete, Resistência dos Materiais, Educação em Engenharia 


\section{INTRODUÇÃ̃}

Para os discentes de engenharia civil, existe uma grande necessidade de um conhecimento estruturado e eficiente sobre diversos assuntos na área da construção civil, inclusive na construção de pontes e viadutos.

É necessário, criar mecanismos que promovam a interação da teoria e prática, a fim de promover um graduando mais preparado e consciente para o mercado de trabalho. Para tanto, a ponte de macarrão surge como uma forma de proporcionar essa interação de unificar ambas as aprendizagens, levando o aprendizado básico do curso à aplicação em uma área de estruturas, as quais possuem forma de treliça (GONZÁLEZ, MORSCH, MASUERO, 2005).

A confecção de pontes de macarrão trata-se de uma atividade realizada em inúmeras instituições brasileiras e no exterior. A primeira instituição a realizar esse projeto foi a Okaganan College em Colúmbia Britânica. Já no Brasil, iniciou-se na Universidade Federal do Rio Grande do Sul sendo, posteriormente, seguido por outras universidades e institutos.

As pontes de macarrão se tornaram eficientes no ensino da área de estruturas visto que os graduandos necessitam realizar testes de tração e compressão e determinar as tensões das barras tanto como as reações que ocorrem nos apoios da ponte. Além disso, utilizou-se o software FTOOL, para o levantamento de cálculos necessários para a construção da ponte de macarrão. O ciclo PDCA (Planejar, Fazer, Checar, Agir) foi utilizado para o controle e melhoria contínua do processo de elaboração e execução da ponte de espaguete.

Neste trabalho descreve-se um caso de sucesso de uma proposta realizada na disciplina de Resistência dos Materiais e tem como objetivo principal construir uma ponte de espaguete que suporte uma carga de $100 \mathrm{kgf}$ obedecendo as normas estipuladas para a competição na Universidade de Vassouras.

\section{FUNDAMENTAÇÃO TEÓRICA}

\subsection{FTOOL}

O FTOOL é um programa que se destina ao ensino do comportamento estrutural de pórticos planos, ocupando um espaço pouco explorado por programas educativos, que se preocupam mais com o ensino das técnicas numéricas de análise, ou por versões educacionais de programas comerciais, mais preocupados em introduzir os estudantes às suas interfaces. Seu objetivo básico é motivar o aluno para aprender o comportamento estrutural (MARTHA, 2021).

Segundo Martha (2021), a experiência de ensino nesta área, têm mostrado que o processo de aprendizado dos métodos de análise estrutural, não são eficientes sem os parâmetros do comportamento estrutural. É muito difícil motivar o aluno nos padrões convencionais, sendo necessário unir teoria e prática como métodos de análise para compreensão do comportamento das estruturas. O processo de aprendizado dos métodos de análise melhoraria significativamente através da interação simultânea entre teoria e prática.

O FTOOL é um software de cálculo estrutural que se destaca pela sua simplicidade e praticidade de manuseio. Permite executar cálculos de estruturas planas de forma rápida e bastante intuitiva, 
evitando a necessidade de recorrer a programas mais complexos. Além disso, o software é gratuito e tem uma forte componente educativa. O software foi desenvolvido pelo professor Luiz Fernando Martha, da Pontifícia Universidade Católica do Rio de Janeiro (PUC-Rio), e está disponível em Martha (2021).

\subsection{PDCA}

Seguindo o conceito de Mattos (2010, pp. 40 - 41) o método PDCA trata didaticamente o processo de melhoria contínua como uma sequência de três passos em um ciclo: aproveitado o máximo dos dados disponíveis para seu desenvolvimento sendo eles de equipes, de orçamento, planos de atuação, tendo a certeza de um planejamento como um compromisso geral e não como missão de uma área técnica; procurando uma execução de uma obra como planejamento, pois nem sempre seu cronograma de obras tem seus objetivos alcançados, tornando necessária uma nova aferição do que foi realizado. Com isso pode ser apropriado índices de campo e propriedade das equipes avaliando seus desvios inerentes a seu planejamento; e por fim uma revisão do seu planejamento dando a ele um novo direcionamento fazendo o gerente retomar sua obra no seu eixo.

Para Couto e Marrash (2012, p. 2) o PDCA nas organizações obtêm resultados contrários, com extensos e volumosos planos tendo como base os procedimentos seguidos na etapa "P" do ciclo PDCA que determina aonde se quer chegar impondo um planejamento eficaz, atingindo um caminho para uma situação desejada, na sua implementação a prática do " $\mathrm{D}$ " trazendo a incerteza da realização de uma atividade importante, pois através de auditorias é encontrado um grande número de atividades fora do seu procedimento, seguindo a etapa "C" identificando algo que não está saindo conforme o planejado. Por fim a etapa "A" responsável para fechar o ciclo PDCA, tão pouco praticada, mas através de ações convincentes e com base nos insucessos nas etapas anteriores garantindo problemas decorrentes dando sentido a um ciclo de melhoria contínua de um determinado processo.

Para Costa (2007, p. 265) o conceito da metodologia do PDCA não consiste somente na implantação das mudanças estratégicas, mas também organizar as melhorias sucessíveis em círculos, composto de quatro fases conforme o descrito no Quadro 1:

\section{Quadro 1 - Etapas do Ciclo PDCA}

\begin{tabular}{|c|l|}
\hline P & $\begin{array}{l}\text { Planejar (Plan) - esta fase parte da preexistência de descrição e entendimento básico do que se } \\
\text { pretende com todo processo. Consiste em definir as ações necessárias, dimensionar os recursos e } \\
\text { condições, identificar as dependências e as implicações, atribuir às responsabilidades especificar o } \\
\text { processo de medição do desempenho e dos resultados esperados. Esta fase é considerada concluida } \\
\text { quando um plano suficientemente detalhado para suportar a execução está propondo e aprovando } \\
\text { para implantação. É nesta fase que se elegem os itens prioritários para implantação. }\end{array}$ \\
\hline D & $\begin{array}{l}\text { Executar - (Do) - execução das ações determinadas no plano, desde a obtenção de recursos e } \\
\text { condições até a implantação do processo de medição e controle. Seu resultado é um conjunto de } \\
\text { sistemas, processos, equipamentos ou que mais tenha sido objetivado no plano, devidamente } \\
\text { implementado e em condições de ser operadoe de produzir os efeitos desejados. }\end{array}$ \\
\hline C & $\begin{array}{l}\text { Verificar ou controlar (Control/Check) - mais do que se medir, implica assegurar que o processo tenha } \\
\text { sido executado mediante observação cuidadosa de seu desempenho planejado na fase P. para isso, } \\
\text { usam-se relatórios de acompanhamento e de desvios, mostrando o atendimento ou não dos } \\
\text { parâmetros de controle estabelecidos. }\end{array}$ \\
\hline A & $\begin{array}{l}\text { Atuar (Act) - na verdade, mais apropriadamente, deveríamos denominar esta fase por "como } \\
\text { aprender com erros e acertos", pois ela é a utilização prática dos resultados do processo, bons ou } \\
\text { maus, para serem introjetados na cultura e nos métodos e sistemas da organização. Assim, a fase } \\
\text { anterior (verificar ou controlar) duas conclusões básicas podem decorrer: ou tudo correu bem, ou } \\
\text { houve problemas. Na primeira hipótese, mais favorável o processo delineado experimentalmente no } \\
\text { planejamento e que foi bem-sucedido deve ser institucionalizado e transformado em padrão para o } \\
\text { futuro. As pessoas precisam ser treinadas ou educadas para agir daquela maneira que deu certo, } \\
\text { seguindo-se, em um novo ciclo, as fases de planejar, executar, verificar e atuar. Isso implica que a } \\
\text { organização aprende como que deu certo. }\end{array}$ \\
\hline
\end{tabular}

Fonte: Adaptado de Costa (2007, p. 266) 
Dentro da aplicação da ferramenta do PDCA várias outras ferramentas dão suporte ao processo, tal como a matriz GUT auxiliará na etapa de planejamento do ciclo do PDCA, permitindo a identificação das causas para a elaboração do plano de ação. Segundo Bond, Busse e Pustilnick (2012, p. 69) a matriz GUT advém da sigla gravidade, urgência e tendência, estabelecendo prioridades com objetivo de eliminar problemas de grandes quantidades relacionados entre si. Com objetivo de saber a gravidade de um problema o GUT identifica seu grau de urgência, como o problema pode ser resolvido e até onde o mesmo pode piorar se nenhuma providência for tomada.

Para auxiliar na identificação das causas, o diagrama de Ishikawa se apresenta como uma ferramenta válida. Segundo Mello (2011, p. 22) o diagrama de Ishikawa é uma ferramenta que serve para identificar as causas de um desvio da qualidade que pode ser denominado como diagrama de causas e efeito ou espinha de peixe, na construção do diagrama é utilizado os $6 \mathrm{Ms}$ como as principais causas dos problemas, sendo eles, a mão de obra, os materiais com seus componentes, as máquinas e equipamentos, os métodos, o meio ambiente e a medição. É válido lembrar que nem sempre é necessária a citação dos seis elementos que compõem o diagrama, podendo o mesmo ter uma solução mais simplificada.

Outra ferramenta relevante no processo de aplicação do PDCA é os cinco porquês, segundo Seleme e Stadler (2012, p. 44) os cinco porquês podem auxiliar na identificação da causa da problemática estudada. A técnica tem como objetivo identificar a verdadeira causa do problema fazendo a sistemática de uma simples pergunta (porquê) propondo soluções cabíveis. Não se torna necessário o uso dos cinco porquês, pois ele pode ser uma natureza simples e de fácil solução, podendo então ser solucionada com mais antecedência.

Para a elaboração do plano de ação que suportará a execução da etapa DO o método 5W2H é amplamente difundido tanto no meio acadêmico quanto no meio organizacional, segundo Custodio (2015, p. 32), a metodologia definida do 5W2H que teve origem nos Estados Unidos define um conjunto de perguntas para melhor eficácia da solução de um problema esta técnica tem por finalidade simplificar o entendimento para a solução de problemas identificando onde deve ser trabalhado. Esta nomenclatura vem do inglês: What (o quê); Why (por que); Where (onde); When (quando); Who (quem); How (como); How much (quanto custa).

\subsection{A Competição}

A Universidade de Vassouras, por meio da disciplina de Resistência de Materiais, vem realizando a Competição da Ponte de Macarrão. O objetivo principal do trabalho proposto é motivar nos alunos o desenvolvimento de habilidades que lhes permitam aplicar conhecimentos básicos adquiridos nas disciplinas Mecânica Básica e Isostática, que de forma interdisciplinar com a disciplina Resistência dos Materiais e Mecânica dos Sólidos, proporcionam ao aluno condições de desenvolver projetos como a ponte de espaguete.

A competição tem sido realizada em várias instituições do país, com o intuito de proporcionar aos seus alunos oportunidades lúdicas de aprendizagem. É uma competição diferente, pois estimula o discente a trabalhar em equipe e aprender a dimensionar uma ponte treliçada de macarrão. Cada competição abrange um edital onde são especificados parâmetros, tais como: tipo do macarrão a ser usado, colas permitidas, materiais para os apoios e a espessura da barra de aço que deve ser colocada no meio da estrutura (onde serão colocados os pesos no dia da competição).

A competição apresenta muitos pontos positivos com relação ao aprendizado que os alunos podem adquirir participando da mesma. Alguns conhecimentos da área de Engenharia Civil são essenciais para a execução do projeto, tais como: Resistência dos Materiais, Pontes, Desenho Técnico (para fazer o desenho do projeto), Cálculo Estrutural, além de ensinar ao aluno a pensar e trabalhar em grupo, executar um projeto, calcular, montar e fazer o acabamento e estética da ponte. Ao 
participar de várias competições ao longo do curso, o aluno passa a ter uma análise mais crítica sobre o próprio projeto, passa a identificar as possíveis falhas e erros, aprende uma maneira nova de executar a montagem e a colagem das barras, o grupo redimensiona o projeto para melhorar a estimativa de carga. O aluno e o grupo adquirem muito conhecimento na área. Na Universidade de Vassouras, em específico, o edital já traz as especificações das dimensões e do limite de peso próprio que a ponte deve ter.

\subsection{Importância do Estudo do Modelo Reduzido no Ensino da Engenharia}

É imprescindível que os profissionais técnicos envolvidos com projetos estruturais, tenham a habilidade de constatar e abarcar o comportamento das estruturas em suas variadas hipóteses de combinações das ações e carregamentos, intrínsecas e extrínsecas à estrutura (HILSON, 1972). Para esse fim, na graduação é essencialmente que os alunos desenvolvam a sapiência espacial intuitiva, para entender o comportamento micro e macro de uma determinada estrutura, desde a primeira fase no parecer Estrutural até a execução da obra, a fim de delinear o projeto arquitetônico associando conhecimento técnico com a importância da otimização ao forçar a estrutura, buscando além obter os aspectos harmoniosos, assegurar maior precisão na apreciação das condições de segurança e viabilidade econômica do empreendimento.

A aplicação de modelos reduzidos qualitativos empregados na instrução do comportamento de estruturas é uma atividade com o intuito de complementar o que vem sendo desenvolvida há mais de sete décadas, como um instrumento didático auxiliador na aprendizagem. Segundo HARRIS et al. (1999) apud OLIVEIRA (2008), um dos primeiros a utilizar modelos estruturais para demonstrações em sala de aula foi RATHBUN, que em 1934 utilizou blocos de madeira presos por arames para demonstrar o comportamento de um arco. Porém, segundo TEIXEIRA (2016), atualmente no "ensino de engenharia, a exploração desta vertente intuitiva é deixada de certa forma para segundo plano. A exposição dos conceitos estruturais é feita quase exclusivamente por via analítica, menosprezando noções qualitativas", o que dificulta a aprendizagem na compreensão dos conceitos teóricos.

Contudo, devemos render uma homenagem aos docentes que dedicam seu tempo para pesquisas acadêmicas. As pesquisas contemporânea tem defendido a importância da análise visual no comportamento de estruturas, através de modelos reduzidos qualitativos, como instrumento de auxílio no ensino/aprendizagem nos cursos de Engenharia e de Arquitetura, citando-se como exemplos: HILSON (1972); SANTOS (1983); REBELLO (1992); SCHWARK (1996); RODRIGUES e HERMIDA (2006); OLIVEIRA (2008); SARAMAGO (2011); TEIXEIRA (2016), dentre outros.

Segundo (PRAVIA, 1995), com o intuito de aprimorar a didática de ensino nas disciplinas de aço e madeira, aplicou trabalhos com construções de modelos reduzidos, como pode averiguar na figura 1, com a finalidade de demonstrar o comportamento de sistemas de coberturas em estruturas de madeira, e relata que os resultados foram excelentes dados as respostas na motivação dos alunos ao utilizar tais modelos como aprendizado prático associado ao conhecimento teórico. 


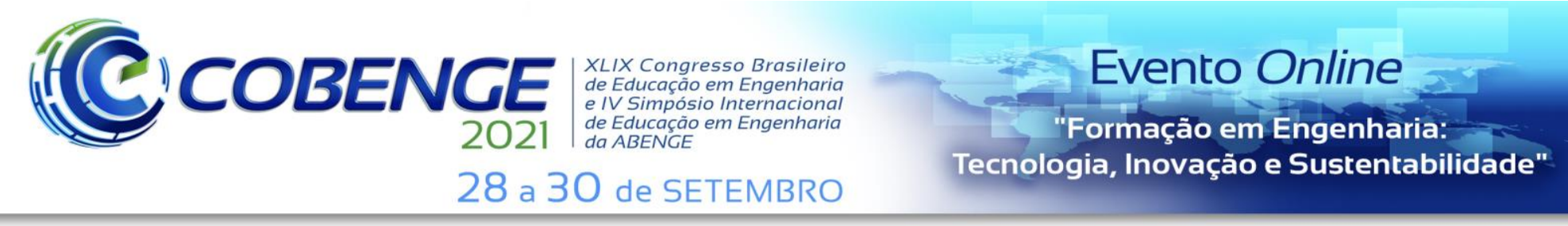

Figura 1: Modelos qualitativos de estruturas: (a) Sistema de cobertura em madeira com treliças howe

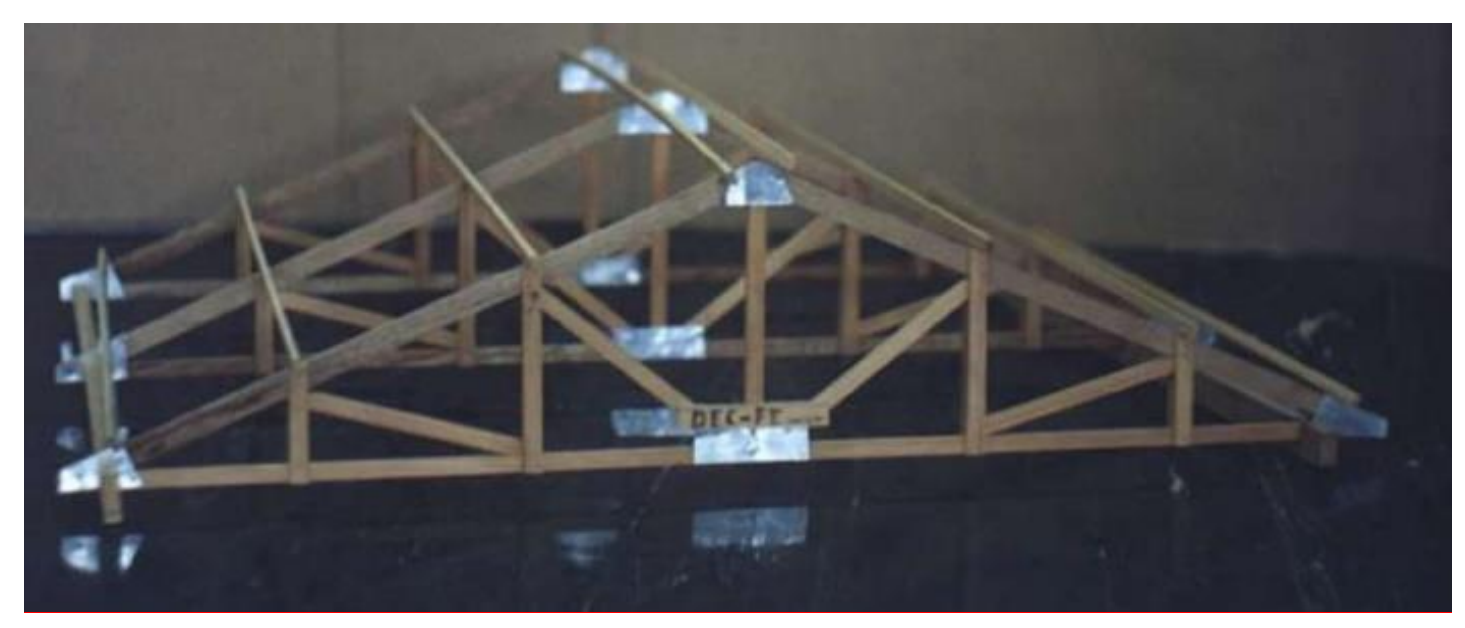

Fonte: PRAVIA,1995

DUPLAT et al. (2008), realizam um trabalho de estudo de ponte treliçada do tipo Warren Modificada, com banzos paralelos, utilizando palitos de picolé e cola, onde os objetivos foram além da construção a realização de testes de carga, como podemos evidenciara na figura 2.

Figura 2: Modelo de ponte treliçada do tipo Warren Modificada construído com palitos de picolé.

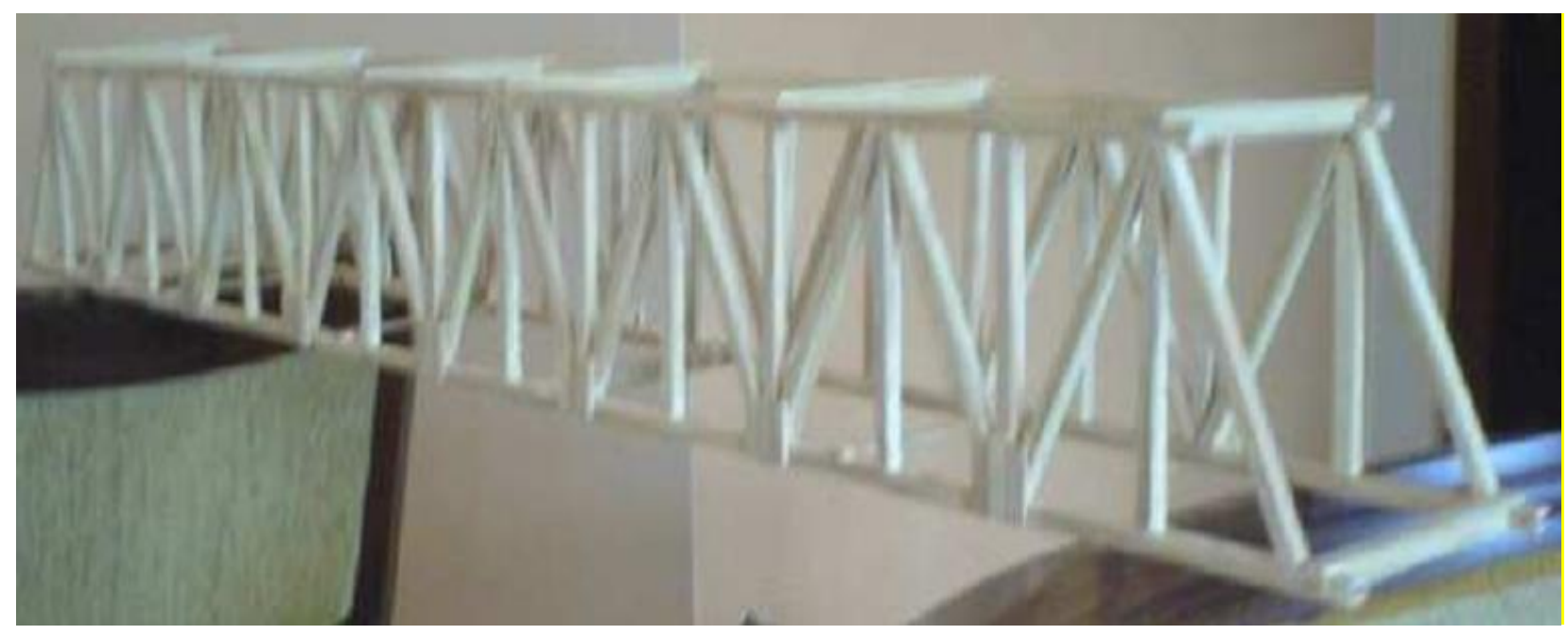

Fonte: DUPLAT et al., 2008

Segundo (REBELLO, 2000), no estudo das estruturas existem duas vertentes que devem ser seguidas, para que o aluno adquira a experiência, fundamental para conceber adequadamente uma estrutura: a da percepção intuitiva e a do conhecimento teórico de cálculo. Para isso, o docente deve desenvolver os conhecimentos teóricos associados ao conhecimento prático, buscando sempre a interação da teoria com a prática 


\section{METODOLOGIA UTILIZADA}

De acordo com Mascarenhas (2012, p. 43), os métodos de pesquisa e investigação são divididos de acordo com; as bases lógicas; a abordagem dada ao problema; o objetivo da pesquisa; e segundo o procedimento técnico utilizado. Para a pesquisa em questão foi adotado como base lógica de investigação a dedução, a partir de recursos lógicos- discursivos para atingir seus objetivos. Segundo Lakatos e Marconi (2010, p. 46), os métodos científicos objetivam a verdade e utilizam a comprovação de hipóteses para chegar a conclusões objetivas, através da observação sistemática do fenômeno estudado e da teoria científica.

De acordo com Matias-Pereira (2012, p. 83), em um sentido holístico os métodos se caracterizam por quantitativos e qualitativos, em que nos métodos qualitativos os dados são analisados de forma indutiva e descritiva não podendo ser quantificada. Já o método quantitativo utiliza métodos estatísticos para análise e tratamento dos dados.

Portanto este estudo utilizará com procedimento técnico o estudo de caso, que permitirá análise de um efeito e em um contexto fundamentado a partir dos conceitos examinados. Neste sentido, essa pesquisa se caracteriza tanto como qualitativa, quanto também aborda aspectos quantitativos, utilizando dados descritivos para análise e discussão dos resultados obtidos.

\section{ANÁLISE E DISCUSSÃO DOS RESULTADOS OBTIDOS}

Os resultados obtidos descrevem os métodos realizados em cada teste executado, obtendo resultados diferentes até a conclusão desejada conforme o projeto desenvolvido. Esses resultados só foram possíveis através da utilização desta poderosa ferramenta de análise de processos, conhecida como PDCA.

$\mathrm{Na}$ primeira etapa dos testes, foram realizados setenta dias de estudos e cálculos para dimensionamento da ponte de espaguete, foram utilizadas dimensões intermediárias, a fim de diminuirmos os nós, porém contamos com emendas para completarmos as barras, o resultado do primeiro teste foi muito ruim, sendo alcançado apenas $2 \mathrm{kgf}$. As figuras 3, 4, e 5 mostram partes deste processo. A Figura 3 mostra a ponte concluída para o teste de carga, a figura 4 apresenta o momento da pesagem e a figura 5 temos a demonstração do teste de carga.

Com este resultado, iniciamos novos estudos, aplicamos o PDCA como alternativa para minimizarmos alguns problemas, assim, foi iniciada a segunda etapa dos testes.

Figura 03 - Ponte construída em casa

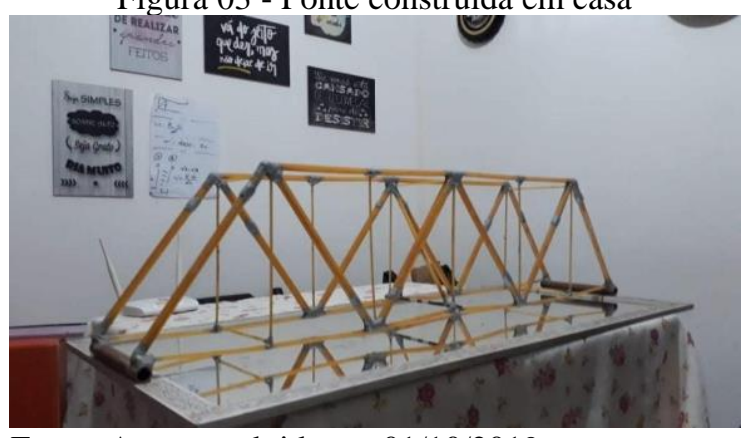

Fonte: Autores, obtida em 01/10/2019
Figura 04 - Pesagem da ponte

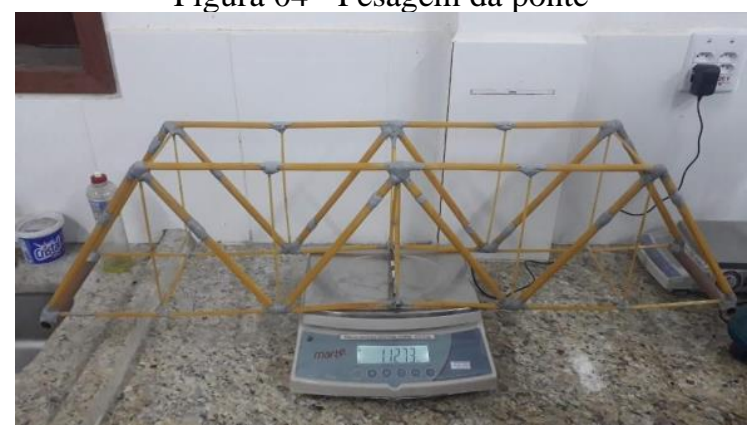

Fonte: Autores, obtida em 01/10/2019 
Figura 05 - Teste de carga

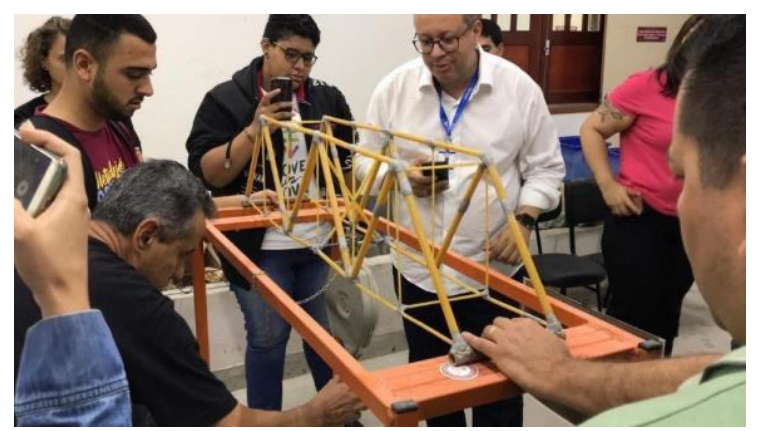

Fonte: Autores, obtida em 01/10/2019

$\mathrm{Na}$ segunda etapa dos testes, as dimensões das barras foram aumentadas, como podemos verificar nas figuras 06 e 07, o resultado foi melhor que o primeiro teste de compressão, resistindo há 8 kgf. Podemos concluir que ao aumentar o comprimento das barras, já identificamos a flambagem das mesmas, como podemos observar nas figuras 06, 07 e 08. Novamente foi aplicado o PDCA, para podermos observar onde estava a deficiência do projeto.

Figura 06 - Construção da ponte de espaguete

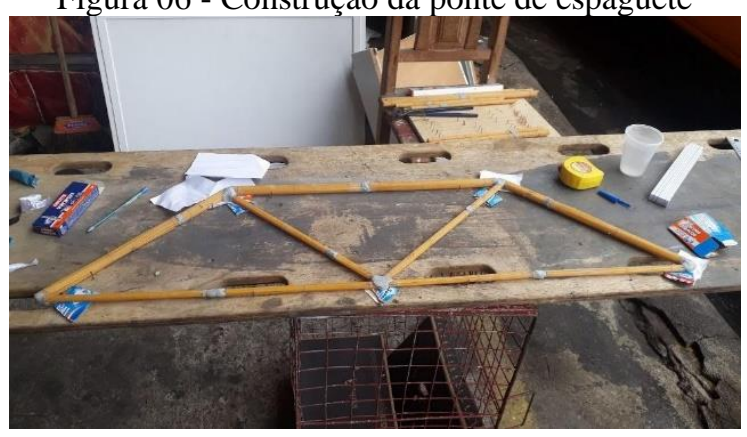

Fonte: Autores, obtida em 13/10/2019
Figura 07 - Ponte pronta para teste de compressão

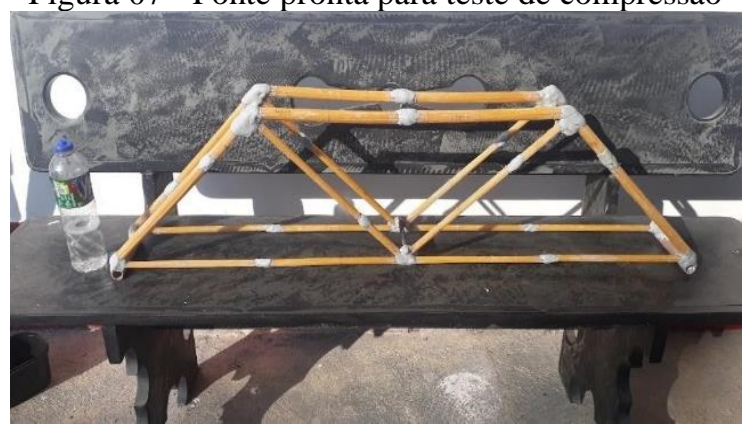

Fonte: Autores, obtida em 13/10/2019

Figura 08 - Pesagem da ponte

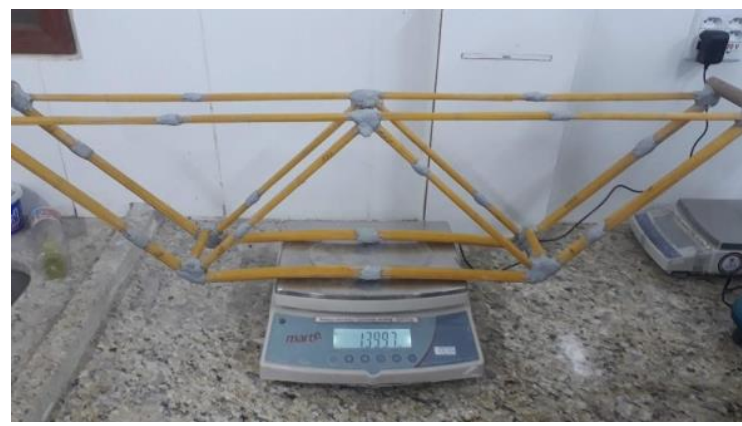

Fonte: Autores, obtida em 13/10/2019

$\mathrm{Na}$ terceira etapa de testes os comprimentos das barras foram dimensionados de acordo com o comprimento do espaguete, minimizando assim emendas desnecessárias que prejudicaram os testes anteriores, nas figuras 09 e 10 podemos evidenciar uma simetria e harmonia melhor nas treliças deste modelo. utilizamos nesta etapa além do durepox usamos uma resina acrílica. No terceiro teste o resultado melhorou significativamente, elevando o teste de carga para $58 \mathrm{kgf}$. Após este teste, 


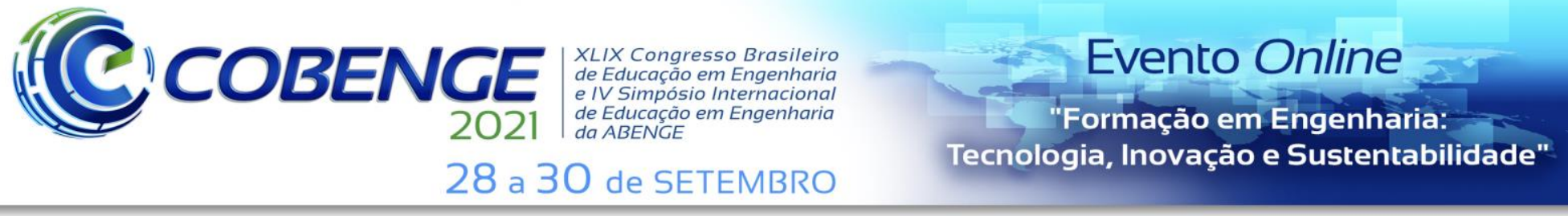

verificou-se ao diminuirmos as emendas, buscando uniformidade no projeto, buscando um resultado melhor e eficiente.

Figura 09 - Pesagem da ponte

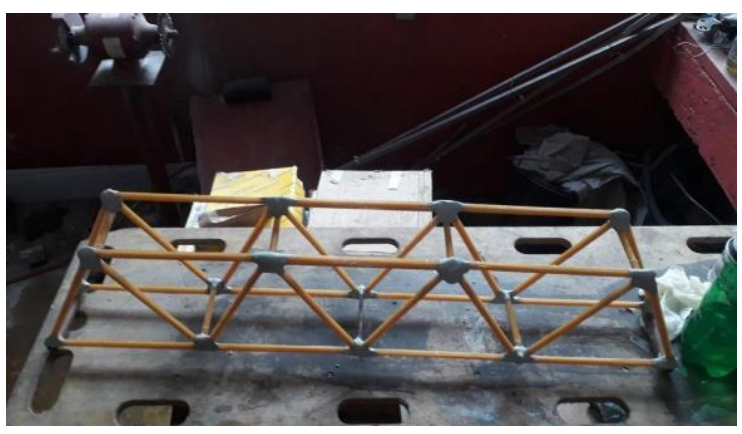

Fonte: Autores, obtida em 25/10/2019
Figura 10 - Pesagem da ponte

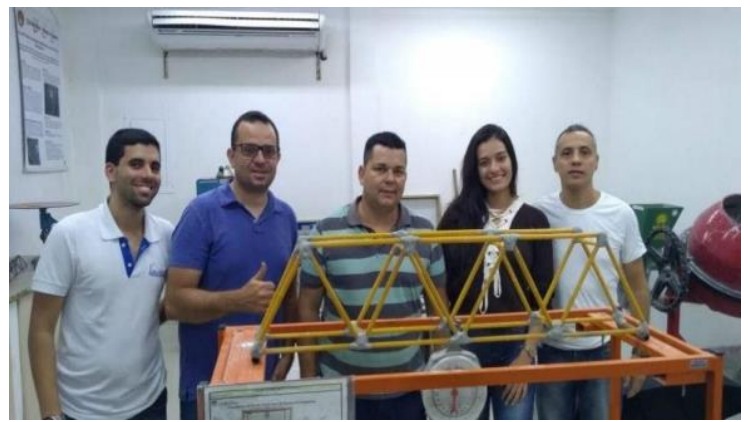

Fonte: Autores, obtida em 25/10/2019

$\mathrm{Na}$ quarta etapa, a competição propriamente dita, foram realizados levantamentos e comparações das etapas anteriores, aplicando novamente o PDCA, com finalidade de avaliar e nortear as novas tomadas de decisão. Nesta etapa final foram utilizados durepox, resina acrílica vitrificada e araldite, visando uma melhor performance do projeto em estudo. As figuras 11, 12, 13, 14, 15 e 16 demonstram a melhoria e exatidão na execução do trabalho final. O trabalho em questão foi dimensionado e executado em dois dias no laboratório da Universidade de Vassouras, buscando utilizar todas as orientações ministradas nas aulas de resistência dos materiais. No teste de compressão a ponte resistiu a $100 \mathrm{kgf}$ conforme projeto, sendo assim superando todas as expectativas iniciais.

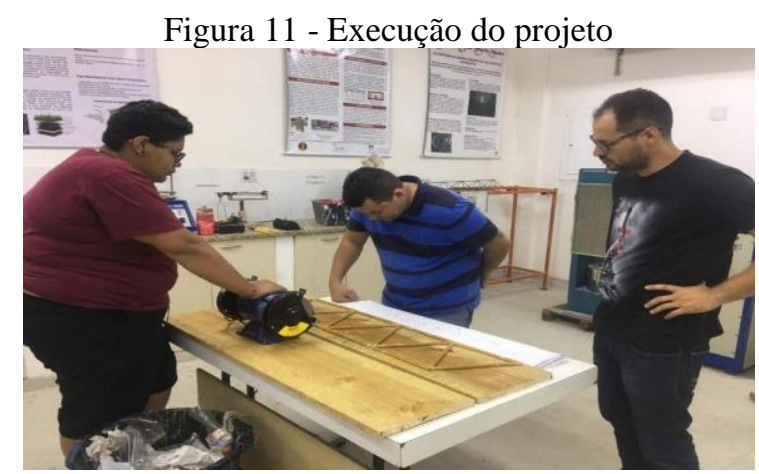

Fonte: Autores, obtida em 27/11/2019

Figura 13 - Projeto pronto

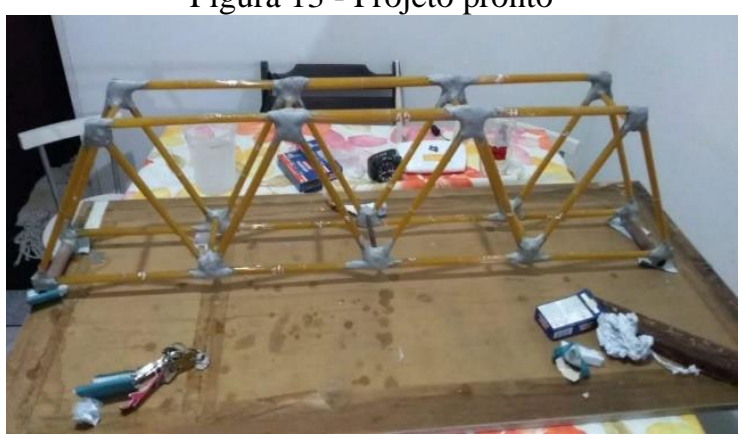

Fonte: Autores, obtida em 27/11/2019
Figura 12 - Execução do projeto

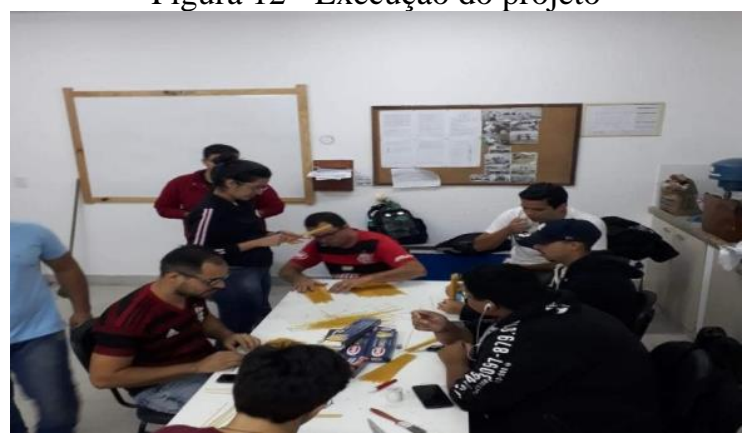

Fonte: Autores, obtida em 27/11/2019

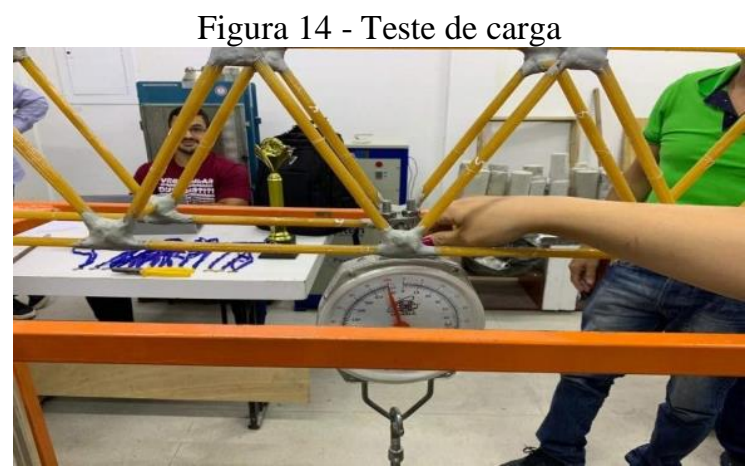

Fonte: Autores, obtida em 28/11/2019 
Figura 15 - Ponte após o teste de compressão

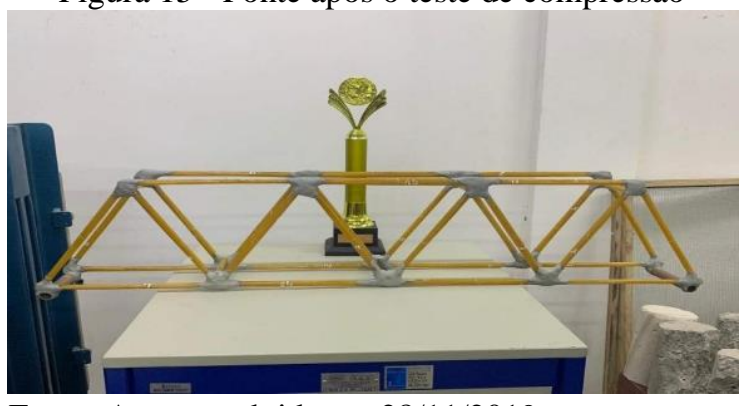

Fonte: Autores, obtida em 28/11/2019
Figura 16 - Equipe que elaborou e executou o projeto

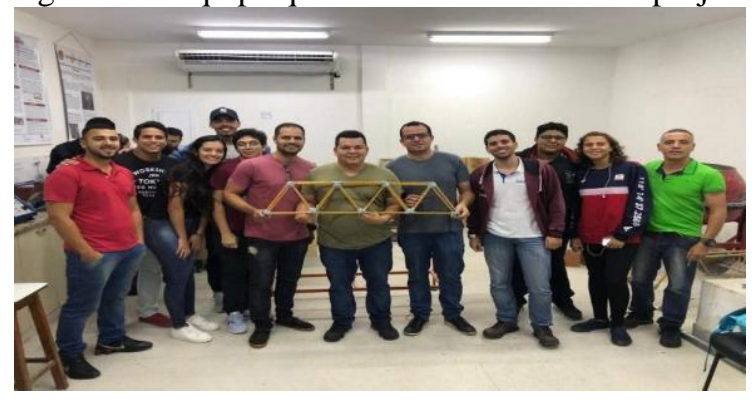

Fonte: Autores, obtida em 28/11/2019

O gráfico 01 evidencia a evolução da resistência dos testes de carga realizados no laboratório da Universidade de Vassouras, o método PDCA foi determinante na obtenção dos dados e tomada de decisões que permitiram a evolução dos resultados obtidos.

Gráfico 01 - Evolução da carga em etapa

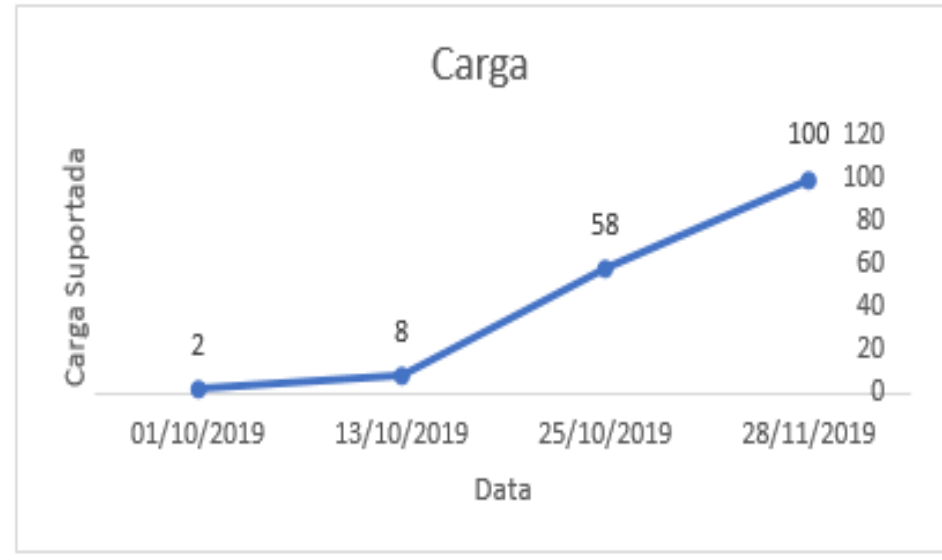

Fonte: Autores

\section{CONCLUSÃO}

Neste contexto, a ferramenta PDCA pode direcionar na melhoria da elaboração e execução de projetos, visando a diminuição das perdas e buscando uma maior precisão, utilizando a melhoria constante como um dos parâmetros para assegurar a satisfação dos clientes e alta qualidade.

Ao término da pesquisa, pôde-se concluir que o propósito inicial que era observar de que maneira a ferramenta PDCA poderia colaborar para a melhoria dos processos foi alcançado, ficando como resultado um mecanismo que enriqueceu o processo de execução e trouxe ganhos significativos aos procedimentos e processos de um modo geral, sendo assim, a ferramenta PDCA foi de extrema importância para comprovação e aplicação da solução final, de maneira eficaz para a melhoria continuada dentro do escopo do projeto sugerido pelo grupo foi essencial para a diminuição de perdas e atingir melhores resultados na execução.

Recomendam-se futuras investigações no setor, que fomentem a aplicabilidade da ferramenta PDCA em outros procedimentos, tanto indústrias, comerciais, quanto de outras áreas de atividade, pois apesar da vasta literatura e obras acadêmicas na área, a aplicabilidade da ferramenta se mostra extensa e flexível. 
Agradecimentos

O último autor agradece ao CNPq pelo apoio recebido no projeto 315216/2018-2.

\section{REFERÊNCIAS}

BOND, M. T., BUSSE, A., \& PUSTILNICK, R. Qualidade total: o que é e como alcançar. Curitiba: Intersaberes, 2012

COSTA, E. A. Gestão estratégica: da empresa que temos da empresa que queremos (2a ed). São Paulo: Saraiva, 2007.

COUTO, B. A., \& MARASH, I. R. Gestão por processos: em sistemas de gestão da qualidade. Rio de Janeiro: Qualitymark, 2012.

CUSTODIO, M. F. Gestão da qualidade e produtividade. São Paulo: Pearson, 2015.

DUPLAT, D. $\mathrm{N}$ et al. Estudo de ponte articulada do tipo Warren Modificada utilizando palitos

de picolé. Trabalho de Resistência dos Materiais EM423, Engenharia Mecânica, UNICAMP. Campinas, 2008.

GONZÁLEZ, L. A. S.; MORSCH, I. B.; MASUERO J. R. Didactic Games in Engineering Teaching - Case: Spaghetti Bridges Design and Building Contest. Ouro Preto: 18th International Congress of Mechanical Engineering, 2005. Disponível em: Acesso em: 15/08/2019

HARRIS, H. G.; SABINS, G. M. Structural modeling and experimental techniques. 2nd ed. CRC Press LLC. Florida, 1999.

HILSON, B. Basic structural behaviour via models. Crosby Lockwood. London, 1972.

LAKATOS, E. M., \& Marconi, M. A. Metodologia científica (5a ed.). São Paulo: Atlas, 2010.

MARTHA, L. F. Manual on-line da versão 2.11. Disponível em: <http://web.tecgraf.pucrio.br/FTOOL/manual/>. Acesso em: 03/04/2021.

MASCARENHAS, S. A. Metodologia científica. São Paulo: Pearson, 2012.

MATIAS-PEREIRA, J. Manual de metodologia da pesquisa científica (3a ed.). São Paulo: Atlas, 2012.

MATTOS, A. D. Planejamento e controle de obras. São Paulo: Pini. 2010.

MELLO, C. H. P. Gestão da qualidade. São Paulo: Pearson Education, 2011.

OLIVEIRA, M. S. Modelo estrutural qualitativo para pré-avaliação do comportamento de estruturas metálicas. Dissertação (Mestrado) Programa de Pós-graduação em Engenharia Civil, Escola de Minas, Universidade Federal de Ouro Preto. Ouro Preto, 2008.

PRAVIA, Z. M. C. Modelos Reduzidos para o Ensino de Estruturas. In: XXIII Congresso Brasileiro de Ensino de Engenharia, 1995, Recife. ANais, 1995. v. 1.

REBELLO, Y. C. P. Contribuição ao ensino de estruturas nas escolas de arquitetura. Dissertação (Mestrado) Faculdade de Arquitetura e Urbanismo de São Paulo. São Paulo, 1992.

RODRIGUES, P. F. N.; HERMIDA, A. S. Modelagem de elementos básicos de estruturas para a análise qualitativa do comportamento estrutural. Revista de Ciência e Tecnologia, Universidade Iguaçu, V. 6, n. 1, jun. 2006.

SANTOS, J. A. Sobre a concepção, o projeto, a execução e a utilização de modelos físicos quantitativos na engenharia de estruturas. Departamento de Engenharia de Estruturas da Escola Politécnica da Universidade de São Paulo. São Paulo, 1983.

SARAMAGO, R. C. P. Ensino de estruturas nas escolas de Arquitetura no Brasil. Dissertação (Mestrado) Arquitetura e Urbanismo, Escola de Engenharia de São Carlos, Universidade de São Paulo. São Carlos, 2011. 
SCHWARK, M. P. Sugestões para um curso intuitivo de teoria das estruturas. Dissertação (Mestrado) Engenharia, Escola Politécnica da Universidade de São Paulo. São Paulo, 1996.

SELEME, R., \& STADLER, H. Controle da qualidade: as ferramentas essenciais. Curitiba: Intersaberes, 2012.

TEIXEIRA, G. A. S. Análise de estruturas porticadas através de modelação física. Dissertação (Mestrado) Engenharia Civil / Estruturas. Faculdade de Engenharia da Universidade do Porto, 2016.

\title{
USING THE PDCA METHODOLOGY TO IMPROVE THE SPAGHETTI BRIDGE CONSTRUCTION PROCESS
}

\begin{abstract}
This work has the objective of showing experimental results from a research carried out at the University of Vassouras and analyze the results obtained in the elaboration of a spaghetti bridge, using the PDCA method as an instrument of improvement in the elaboration and execution of the project. The evaluation was performed through compression and traction tests in the laboratory of the University of Vassouras. The execution started and during the process several problems were identified both in the materials and in the execution method, where for the materials they were submitted to a quality control being discarded the standardized ones and in the execution, templates were used for the assembly with the pre-established measures by the teacher, during the whole process the improvement was being made observing the result in the stress test and so the whole process was improved, eliminating possible errors until the result defined in the project was acquired.
\end{abstract}

Keywords: PDCA, Spaghetti Bridge, Strength of Materials, Engineering Education 